\title{
Efficacy and Tolerability of Delayed-release Dimethyl Fumarate in Black, Hispanic, and Asian Patients with Relapsing-Remitting Multiple Sclerosis: Post Hoc Integrated Analysis of DEFINE and CONFIRM
}

Robert J. Fox · Ralf Gold · J. Theodore Phillips · Macaulay Okwuokenye •

Annie Zhang · Jing L. Marantz

Received: May 26, 2017 / Published online: August 2, 2017

(C) The Author(s) 2017. This article is an open access publication

\begin{abstract}
Introduction: Clinical course and treatment response may vary according to race/ethnicity in multiple sclerosis (MS) patients. Delayed-release dimethyl fumarate (DMF; also known as gastro-resistant DMF) demonstrated significant efficacy and a favorable benefit-risk profile in relapsing-remitting MS (RRMS) patients in the 2-year phase III DEFINE/CONFIRM studies.

Methods: In this post hoc analysis of integrated data from DEFINE/CONFIRM, we assessed clinical efficacy and safety/tolerability in black, Hispanic, and Asian patients treated with DMF $240 \mathrm{mg}$ twice daily (approved dosage) or placebo. Eligible patients were $18-55$ years of age
\end{abstract}

Enhanced content To view enhanced content go to http://www.medengine.com/Redeem/ 6DD8F06015440AF3.

R. J. Fox

Cleveland Clinic, Mellen Center for Multiple Sclerosis Treatment and Research, Cleveland, $\mathrm{OH}$, USA

R. Gold

St. Josef Hospital, Ruhr University, Bochum, Germany

J. T. Phillips

Multiple Sclerosis Program, Baylor Institute for Immunology Research, Dallas, TX, USA

M. Okwuokenye $(\bowtie) \cdot$ A. Zhang · J. L. Marantz Biogen, Cambridge, MA, USA

e-mail: macaulay.okwuokenye@biogen.com with an Expanded Disability Status Scale score of $0-5.0$. In the integrated intention-to-treat population, 769 and 771 patients were treated with DMF or placebo, respectively, of whom 10 and 19 were black, 31 and 23 were Hispanic, and 66 and 70 were Asian.

Results: In the black, Hispanic, and Asian subgroups, DMF was associated with lower annualized relapse rates at 2 years compared with placebo [rate ratio $(95 \%$ confidence interval (CI)), 0.05 (0.00-1.07); 0.31 (0.10-0.95); and $0.64(0.30-1.34)$, respectively]. The percentage of black, Hispanic, and Asian patients with 12-week confirmed disability progression was lower with DMF (43\%, 8\%, and 20\%, respectively) compared with placebo [57\%, 30\%, and $25 \%$, respectively; hazard ratio $(95 \% \mathrm{CI}), 0.53$ (0.02-1.39); $0.17 \quad(0.00-0.60) ; \quad$ and 0.71 $(0.32-1.58)$, respectively]. The safety/tolerability profile of DMF was generally consistent with that in the overall population of DEFINE/ CONFIRM. The incidence of adverse events leading to treatment discontinuation in black, Hispanic, and Asian patients was 2/10, 2/31, and $3 / 66$, respectively, with DMF, and $2 / 19$, $1 / 23$, and $8 / 70$, respectively, with placebo.

Conclusion: DMF may be an efficacious treatment with a favorable benefit-risk profile in black, Hispanic, and Asian patients with RRMS. Further clinical studies are needed to characterize differences in MS presentation and treatment outcomes across ethnic and racial groups. Funding: Biogen. 
Trial Registration: DEFINE: ClinicalTrials.gov identifier NCT00420212; CONFIRM ClinicalTrials.gov identifier NCT00451451.

Keywords: Delayed-release dimethyl fumarate; Efficacy; Ethnicity; Race; Relapsing-remitting multiple sclerosis; Tolerability

\section{INTRODUCTION}

Multiple sclerosis (MS) is characterized by complex genetic, autoimmune, and environmental interactions, with noted ethnic, racial, and geographic differences in susceptibility to the disease [1]. There are contrary reports on the incidence rate of MS across ethnic subgroups. While historically the incidence of MS was described as higher in white patients than in black patients [1], a recent study reported that the incidence is higher in black patients than in other ethnic groups in the USA, with a $47 \%$ higher risk compared with white patients and a $50 \%$ and $80 \%$ lower risk for Hispanic and Asian patients, respectively [2]. This difference in reported rates might be explained by the regional environmental effect, which can be specific to the location of the study (Southern California), and the system used (Kaiser Permanente) to evaluate the study cohort [2].

Although data remain limited, studies have also indicated that the clinical course and disability outcomes associated with MS may vary according to ethnicity and race. For example, several retrospective studies have found that black patients with MS may experience a more aggressive and rapidly disabling disease course than white patients [3-5]. Analysis of a large North American MS database found that Hispanic and black patients were younger at diagnosis than white patients, with earlier onset of symptoms [6]; an earlier study had suggested that black patients were more likely to have an older age at disease onset [7]. It has been noted that Asian patients with MS reached the same level of disability 5-10 years earlier than patients from other regions, predominantly driven by progressive course of illness, a history of transverse myelitis, incomplete recovery from the first attack, and abnormal somatosensory evoked potentials [8]. Furthermore, non-white patients with MS may be almost 2.5 times more likely to experience a second MS relapse within the first year from onset compared with white patients [9].

Non-white races and ethnicities have been largely underrepresented in MS clinical trials [10], despite making up more than $27 \%$ of the total population in the USA (including 12.6\% black and $4.8 \%$ Asian patients) [11]. This has made it difficult to evaluate the efficacy and tolerability of treatments in populations that may present with a biologically different form of the disease than in white patients with MS. Small post hoc or retrospective studies have suggested that black patients with MS are less responsive than white patients to treatment with interferon beta-1a [12] or specific disease-modifying therapies in general [13]. However, post hoc analyses of clinical trials evaluating natalizumab and fingolimod have suggested that these agents may be efficacious in Hispanic and African descent patients with MS $[14,15]$.

Delayed-release dimethyl fumarate (DMF; also known as gastro-resistant DMF) is a novel orally administered therapy approved for use in patients with relapsing MS (USA/Australia) $[16,17]$ and relapsing-remitting MS (RRMS; European Union/Canada) $[18,19]$. To date, over 260,000 patients with relapsing forms of MS have been treated with DMF (Biogen, data on file). In two pivotal phase III studies in patients with RRMS (DEFINE and CONFIRM), DMF significantly reduced the annualized relapse rate (ARR), the percentage of patients relapsed (PPR), and the number of lesions on magnetic resonance imaging (MRI) compared with placebo $[20,21]$. The risk of 12-week confirmed disability progression (CDP) over 2 years also was reduced in both studies, reaching statistical significance in DEFINE. An integrated analysis of data from DEFINE and CONFIRM stratified by various demographic and disease characteristics suggested that DMF may be an appropriate treatment choice across a broad range of subgroups of patients with RRMS [22]. Therefore, the purpose of this post hoc analysis of integrated data from the DEFINE and CONFIRM studies was to assess the efficacy and tolerability 
of DMF in RRMS patients by racial group: black, Hispanic, and Asian. Data for DMF $240 \mathrm{mg}$ twice daily (BID; the approved dosage regimen) are reported for this exploratory analysis.

\section{METHODS}

\section{Patients and Study Design}

The methodologies for the phase III international, randomized, double-blind DEFINE (ClinicalTrials.gov identifier NCT00420212) and CONFIRM (ClinicalTrials.gov identifier NCT00451451) studies are similar and have been published previously [20, 21]. In brief, patients 18-55 years of age with RRMS (McDonald criteria), with a score of $0-5.0$ on the Expanded Disability Status Scale (EDSS) [23, 24], and at least one clinically documented relapse in the previous 12 months or at least one gadolinium-enhancing lesion 0-6 weeks before randomization were eligible for study inclusion. Patients were excluded if they had progressive forms of MS or another clinically significant illness.

Patients were randomized to oral administration of DMF $240 \mathrm{mg}$ BID or three times daily (TID) or matching placebo for up to 96 weeks. In CONFIRM only, an additional group of patients was randomized to receive the active reference comparator glatiramer acetate at a dose of $20 \mathrm{mg}$ once daily, administered by subcutaneous injection. Patients receiving glatiramer acetate were aware of their treatment allocation. All raters were blinded to treatment assignment.

Standardized neurologic assessments, including an EDSS assessment, were performed every 12 weeks and at an unscheduled visit at the time of suspected relapse. Relapses were confirmed by an independent neurological evaluation committee. Safety assessments, including the monitoring of laboratory values and collection of adverse events (AEs), were performed every 4 weeks.

Each study was performed at multiple sites across 28 countries. The studies DEFINE and CONFIRM were approved by central and local ethics committees and were conducted in accordance with the International Conference on Harmonisation's guidelines for Good Clinical Practice and the Declaration of Helsinki. All procedures followed were in accordance with the ethical standards of the responsible committee on human experimentation (institutional and national) and with the Helsinki Declaration of 1964, as revised in 2013. All patients provided written informed consent before undergoing any study procedures and re-consent in the event of confirmed relapse or disability progression.

The present analysis was performed only on the DMF $240 \mathrm{mg}$ BID- and placebo-treated patients; the TID subgroup was excluded from this analysis.

\section{Integrated Analysis and Endpoints}

Integrated analyses were preplanned before the unblinding of CONFIRM and were to be conducted only if the DEFINE and CONFIRM studies were similar in terms of several key characteristics [22]. Generally, integrated analyses present several advantages: they allow for evaluation of a greater number of patients than the individual studies, provide a more precise estimate of a therapeutic effect, and facilitate assessment of the consistency of an effect across different subgroups of patients [22].

Efficacy study endpoints analyzed were ARR at 2 years (primary endpoint in CONFIRM and a secondary endpoint in DEFINE), PPR at 2 years (primary endpoint in DEFINE and a secondary endpoint in CONFIRM), and time to 12-week $\mathrm{CDP}$ at 2 years (secondary endpoint in CONFIRM and DEFINE).

In this post hoc integrated analysis, the clinical endpoints were evaluated in black, Hispanic, and Asian patients with RRMS who were assigned to receive DMF $240 \mathrm{mg}$ BID (hereafter referred to as DMF) or placebo in the CONFIRM and DEFINE studies. This represents the approved dosage (240 mg BID) of DMF in all geographic regions in which it is approved. 


\section{Statistical Analysis}

The efficacy and safety analyses were based on all randomized patients who received at least one dose of study drug.

For the Asian patient subgroup, analyses of ARR, PPR, and time to 12-week CDP were conducted using classical methodology as employed in DEFINE and CONFIRM [20, 21]. Briefly, adjusted ARRs, rate ratios, and 95\% confidence intervals (CIs) at 2 years were based on negative binomial regression, adjusted for baseline EDSS score ( $\leq 2.0$ vs. $>2.0$ ), baseline age ( $<40$ vs. $\geq 40$ years), study, region, and number of relapses in the first year before study entry. PPR and CDP were estimated as the cumulative probability of relapse and disability, respectively, from the Kaplan-Meier curve of time to first relapse and time to first 12-week CDP (i.e., Kaplan-Meier product limit estimator). For treatment comparisons, hazard ratios (HRs) and 95\% CIs were based on a stratified Cox proportional hazards model with study as a stratifying variable, adjusted for baseline EDSS score $(\leq 2.0$ vs. $>2.0)$, baseline age $(<40$ vs. $\geq 40$ years), region, and number of relapses in the first year before study entry (for PPR outcomes only).

As a result of the small sample size in the black and Hispanic patient subgroups, for the analyses of ARR, PPR, and time to 12-week CDP at 2 years, Bayesian statistical methods were employed. Because of the sparse frequency of relapse in black patients, the Firth correction for monotonic likelihood $[25,26]$ was applied for the analysis of PPR within this subgroup. An unadjusted Poisson regression model was used for the analysis of ARR in black patients because of convergence issues encountered with the negative binomial regression model. Diffuse, non-informative priors were used for the coefficient of parameters to discount prior information and to reflect objective representation of unavailable prior information on the treatment effects of DMF among the racial subgroups. Specifically, the prior means of the parameter coefficients were either Jeffreys' prior or centered at zero (0) with large variances of $10^{6}$. Convergence was assessed using the Geweke test and other graphic methods. Sensitivity analyses were conducted to assess the robustness of the posterior distribution to change in prior information.

CIs are presented for analyses based on the classical approach, and high probability density credible intervals (Bayesian CIs) of the posterior means of the estimates are presented for the analyses based on Bayesian statistical methods.

\section{RESULTS}

\section{Patients}

The integrated intention-to-treat (ITT) population comprised 1540 patients, including 769 patients treated with DMF $240 \mathrm{mg}$ BID and 771 patients treated with placebo, of whom 10 and 19 were black, 31 and 23 Hispanic, and 66 and 70 Asian (Table 1). In general, the baseline demographic and clinical characteristics were well balanced across the subgroups and between the placebo and DMF treatment arms, with the exception of the percentage of women among Asian patients $(54 \%$ in the placebo group and $73 \%$ in the DMF-treated group).

\section{Efficacy}

ARR was lower in each of the racial subgroups treated with DMF compared with placebo (Figs. 1, 2a). In the black subgroup, the posterior mean of the adjusted ARR (95\% CI; Bayesian CIs) at 2 years for DMF-treated patients was $0.01(0.00-0.29)$ compared with $0.27(0.13-0.55)$ in the placebo group; the rate ratio (95\% CI) was 0.05 (0.00-1.07). In the Hispanic subgroup, the adjusted ARR (95\% CI) for DMF-treated patients was 0.15 (0.04-0.56) compared with $0.49(0.14-1.68)$ in the placebo group; the rate ratio $(95 \% \mathrm{CI})$ was $0.31(0.10-0.95)$. For the Asian subgroup, the adjusted ARR $(95 \% \mathrm{CI})$ in the DMF group was $0.14(0.03-0.66)$ compared with $0.21(0.05-0.93)$ in the placebo group; the rate ratio was 0.64 (0.30-1.34) (Table 2).

The PPR at 2 years was numerically lower with DMF than with placebo in each subgroup (Fig. 2b). Compared with placebo, DMF was associated with a lower risk of MS relapse at 2 years by $93 \%$ [HR (95\% CI), $0.07(0.00-0.33)$ ] in the black subgroup, by $45 \%$ [HR (95\% CI), 
Table 1 Baseline characteristics across black, Hispanic, and Asian patients (integrated analysis of DEFINE and CONFIRM)

\begin{tabular}{|c|c|c|c|c|c|c|c|c|}
\hline \multirow[t]{2}{*}{ Characteristic $^{\mathrm{a}}$} & \multicolumn{2}{|c|}{ Black patients } & \multicolumn{2}{|c|}{ Hispanic patients } & \multicolumn{2}{|c|}{ Asian patients } & \multicolumn{2}{|c|}{ ITT population } \\
\hline & $\begin{array}{l}\text { Placebo } \\
(n=19)\end{array}$ & $\begin{array}{l}\mathrm{DMF}^{\mathrm{b}} \\
(n=10)\end{array}$ & $\begin{array}{l}\text { Placebo } \\
(n=23)\end{array}$ & $\begin{array}{l}\text { DMF }^{\mathbf{b}} \\
(n=31)\end{array}$ & $\begin{array}{l}\text { Placebo } \\
(n=70)\end{array}$ & $\begin{array}{l}\mathrm{DMF}^{\mathrm{b}} \\
(n=66)\end{array}$ & $\begin{array}{l}\text { Placebo } \\
(n=771)\end{array}$ & $\begin{array}{l}\mathrm{DMF}^{\mathrm{b}} \\
(n=769)\end{array}$ \\
\hline Age, years & $37.3(10.2)$ & $39.0(8.7)$ & $32.1(8.2)$ & $31.7(10.3)$ & $34.8(9.8)$ & $34.0(9.1)$ & $37.7(9.2)$ & $37.9(9.2)$ \\
\hline Female, $n(\%)$ & $14(73.7)$ & $7(70.0)$ & $14(60.9)$ & $21(67.7)$ & $38(54.3)$ & $48(72.7)$ & $557(72.0)$ & $541(70)$ \\
\hline $\begin{array}{l}\text { Time since first } \\
\text { MS } \\
\text { symptoms, } \\
\text { years }\end{array}$ & $7.3(8.2)$ & $5.6(6.4)$ & $5.3(4.9)$ & $4.5(4.8)$ & $6.0(4.6)$ & $6.2(4.9)$ & $8.1(6.5)$ & $8.3(6.8)$ \\
\hline $\begin{array}{l}\text { Time since first } \\
\text { MS diagnosis, } \\
\text { years }\end{array}$ & $4.3(7.4)$ & $3.1(3.8)$ & $4.0(4.6)$ & $3.3(5.0)$ & $4.3(4.1)$ & $4.0(3.3)$ & $5.3(5.5)$ & $5.3(5.3)$ \\
\hline $\begin{array}{l}\text { Prior approved } \\
\text { MS } \\
\text { treatment }{ }^{c}, \\
n(\%)\end{array}$ & $11(57.9)$ & $4(40.0)$ & $7(30.4)$ & $10(32.3)$ & $6(8.6)$ & $5(7.6)$ & $95(12)$ & $50(7)$ \\
\hline $\begin{array}{l}\text { Number of } \\
\text { relapses in } \\
\text { prior year }\end{array}$ & $1.4(0.6)$ & $1.8(0.9)$ & $1.5(1.5)$ & $1.4(0.6)$ & $1.6(0.9)$ & $1.4(0.9)$ & $1.3(0.7)$ & $1.3(0.7)$ \\
\hline EDSS score & $2.1(1.0)$ & $1.9(1.6)$ & $2.3(1.4)$ & $2.0(1.1)$ & $2.8(1.1)$ & $3.3(1.1)$ & $2.5(1.2)$ & $2.5(1.3)$ \\
\hline
\end{tabular}

EDSS Expanded Disability Status Scale, $M S$ multiple sclerosis, $S D$ standard deviation

${ }^{a}$ Values are mean $\pm S D$ unless otherwise stated

b DMF denotes delayed-release dimethyl fumarate (DMF; also known as gastro-resistant DMF) at the approved dosage, $240 \mathrm{mg}$ BID

${ }^{c}$ Approved medications for multiple sclerosis included glatiramer acetate, interferon (beta-1a and beta-1b), and natalizumab. Patients may have received more than one prior medication for multiple sclerosis. Patients may also have received other, nonapproved therapies for multiple sclerosis

$0.55(0.07-1.28)]$ in the Hispanic subgroup, and by $34 \%$ [HR (95\% CI), $0.66(0.35-1.24)]$ in the Asian subgroup (Fig. 2b).

The 12-week disability progression HR for the Asian subgroup and the posterior mean of HR (from Bayesian method) for the black and Hispanic subgroups favored DMF treatment, but were not statistically significant (Fig. 2c).

\section{Safety}

The overall incidence of AEs in the black, Hispanic, and Asian subgroups with DMF vs. placebo was $80 \%$ vs. $89 \%, 97 \%$ vs. $96 \%$, and $91 \%$ vs. 91\%, respectively (Table 3). Headache, proteinuria, albumin in the urine, and paresthesia were among the most frequent AEs with DMF treatment in the black subgroup, whereas headache, flushing, nasopharyngitis, and diarrhea were more common in the Hispanic subgroup. Proteinuria, albumin in the urine, and pyrexia were more common in Asian patients. No deaths due to AEs were reported. The incidence of AEs leading to discontinuation of treatment in black, Hispanic, and Asian patients with DMF vs. placebo was 2/10 vs. 2/19 for black 

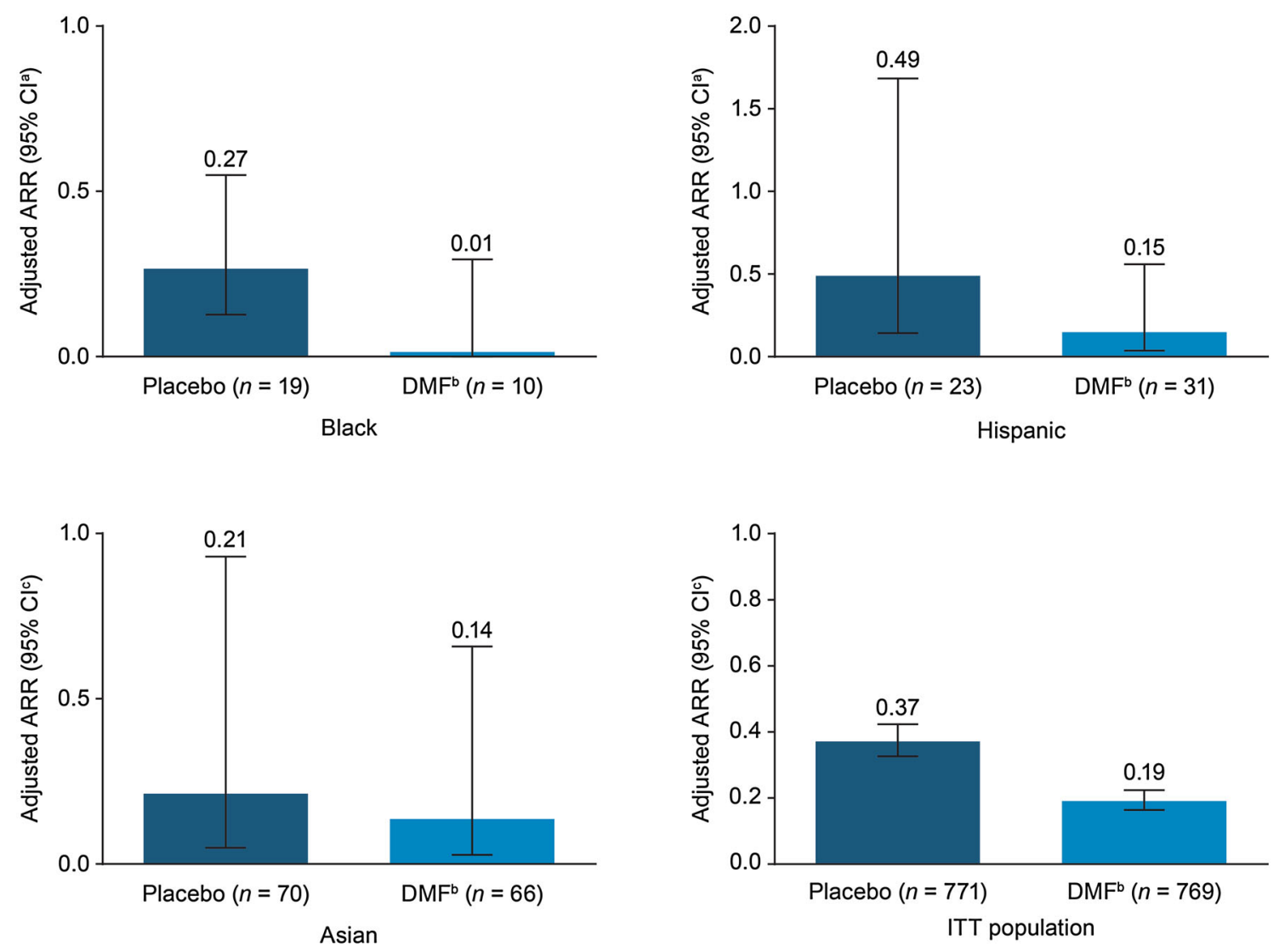

Fig. 1 Adjusted annualized relapse rate (ARR) at 2 years in the intention-to-treat (ITT) population of CONFIRM and DEFINE and across ethnic subgroups (integrated analysis of DEFINE and CONFIRM). ${ }^{\text {a } C I ~ d e n o t e s ~}$

patients, $2 / 31$ vs. 5/23 for Hispanic patients, and $3 / 66$ vs. $8 / 70$ for Asian patients.

\section{DISCUSSION}

In this exploratory analysis we examined the DMF treatment outcomes among black, Hispanic, and Asian patients. This post hoc integrated analysis of data from DEFINE and CONFIRM suggests that DMF at its approved dosage of $240 \mathrm{mg}$ BID may have a significant therapeutic effect in black, Hispanic, and Asian patients with RRMS. Over 2 years, ARR and the risk of relapse with DMF were lower compared with placebo; 12 -week CDP favored DMF treatment in all three races analyzed. No statistically significant differences in the estimated

confidence interval. ${ }^{\mathrm{b}} \mathrm{DMF}$ denotes delayed-release dimethyl fumarate (DMF; also known as gastro-resistant $\mathrm{DMF}) .{ }^{\mathrm{c}} \mathrm{CI}$ denotes credible interval

probability of disability progression were observed across black, Hispanic, and Asian patients. This is likely due to the small sample size and the low rate of disability progression within these groups.

These results are broadly consistent with those obtained in the phase III clinical studies $[20,21]$, and those from a recent retrospective chart-review analysis performed in the USA [27]. In the latter study, the authors concluded that DMF efficacy is independent of the patient's race. The real-world study involved 261 white, 69 black, and 52 Hispanic patients and evaluated ARR, new T2 lesions, and gadolinium-enhancing lesions.

In the integrated analysis of the DEFINE and CONFIRM ITT population treated with DMF $\mathrm{BID}$, there was a relative reduction in ARR of 

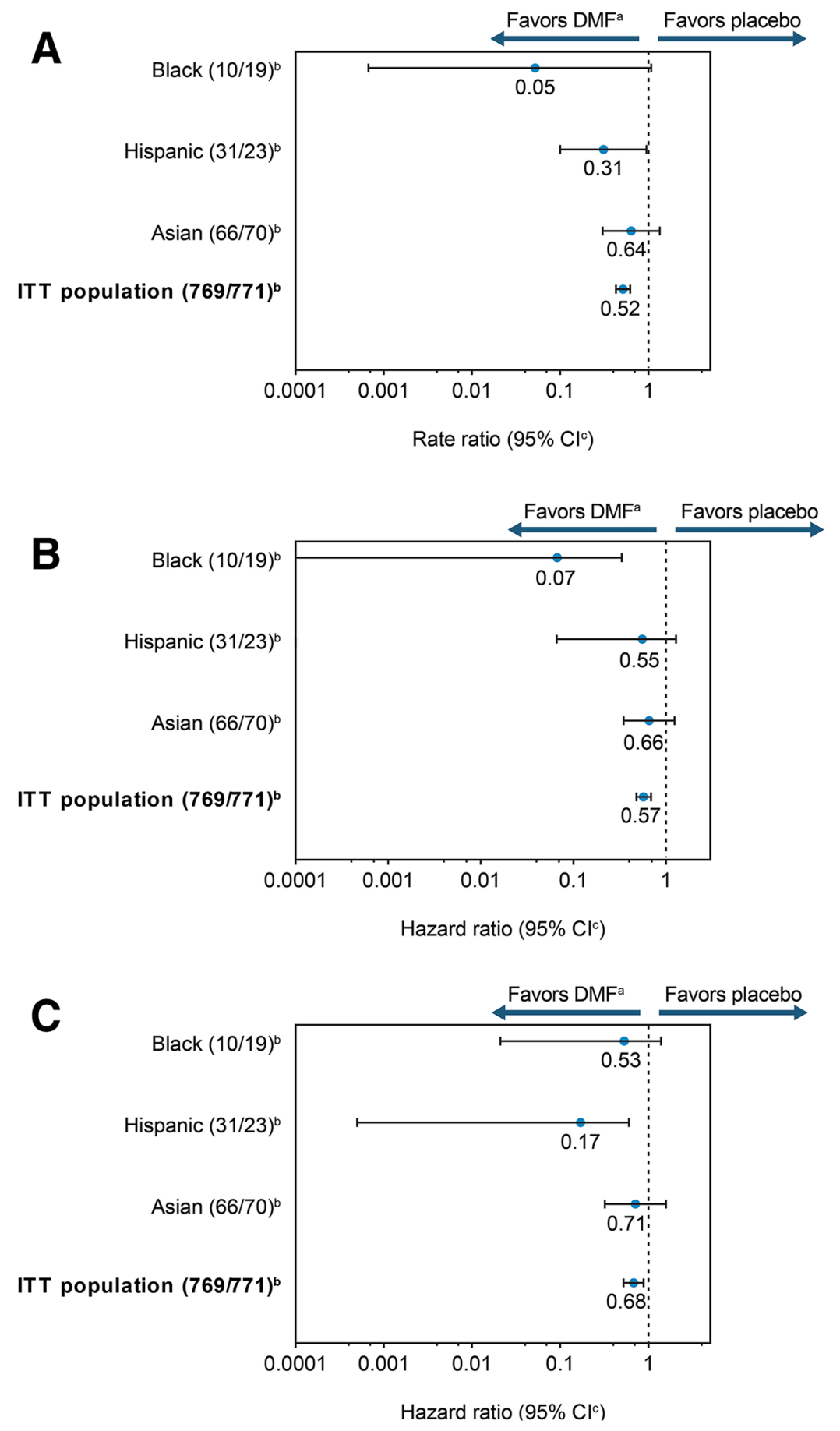

Fig. 2 Clinical efficacy with delayed-release DMF. a Rate ratio for the adjusted annualized relapse rate (ARR) at 2 years. b Hazard ratio for proportion of patients with relapse at 2 years. c Hazard ratio for 12-week confirmed disability progression at 2 years. ${ }^{a} \mathrm{DMF}$ denotes delayed-release dimethyl fumarate (DMF; also known as gastro-resistant DMF) vs. placebo in ethnic subgroups (integrated analysis of DEFINE and CONFIRM). ${ }^{b}$ Number of patients treated with DMF twice daily/placebo. ${ }^{c}$ Intervals represent $95 \%$ confidence intervals for the overall ethnic subgroup and Asian patients with multiple sclerosis (MS) and $95 \%$ credible intervals for black and Hispanic patients with MS. CI confidence/credible interval, ITT intention-to-treat 


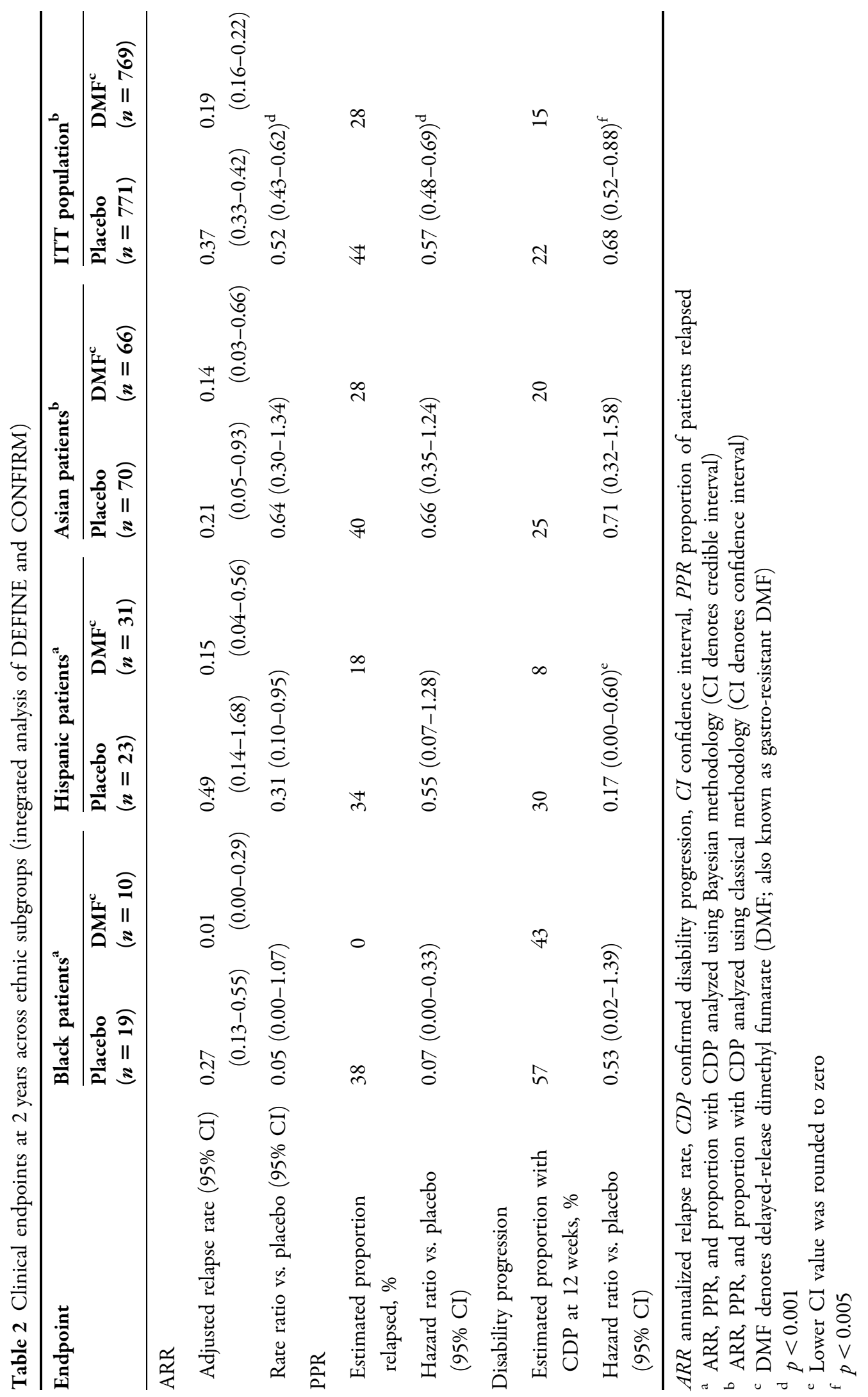


Table 3 AEs reported across black, Hispanic, and Asian patients, and ITT population (integrated analysis of DEFINE and CONFIRM)

\begin{tabular}{|c|c|c|c|c|c|c|c|c|}
\hline \multirow[t]{2}{*}{$\mathrm{AE}, n(\%)$} & \multicolumn{2}{|c|}{ Black patients } & \multicolumn{2}{|c|}{ Hispanic patients } & \multicolumn{2}{|c|}{ Asian patients } & \multicolumn{2}{|c|}{ ITT population } \\
\hline & $\begin{array}{l}\text { Placebo } \\
(n=19)\end{array}$ & $\begin{array}{l}\mathrm{DMF}^{\mathrm{a}} \\
(n=10)\end{array}$ & $\begin{array}{l}\text { Placebo } \\
(n=23)\end{array}$ & $\begin{array}{l}\mathrm{DMF}^{\mathrm{a}} \\
(n=31)\end{array}$ & $\begin{array}{l}\text { Placebo } \\
(n=70)\end{array}$ & $\begin{array}{l}\mathrm{DMF}^{\mathrm{a}} \\
(n=66)\end{array}$ & $\begin{array}{l}\text { Placebo } \\
(n=771)\end{array}$ & $\begin{array}{l}\mathrm{DMF}^{\mathrm{a}} \\
(n=769)\end{array}$ \\
\hline Any AE & $17(89)$ & $8(80)$ & $22(96)$ & $30(97)$ & $64(91)$ & $60(91)$ & $720(93)$ & $733(95)$ \\
\hline \multicolumn{9}{|l|}{ Most common $\mathrm{AEs}^{\mathrm{b}, \mathrm{c}}$} \\
\hline MS relapse & $7(37)$ & $1(10)$ & $7(30)$ & $6(19)$ & $28(40)$ & $19(29)$ & $344(45)$ & $221(29)$ \\
\hline Flushing & 0 & $2(20)$ & $1(4)$ & $6(19)$ & $1(1)$ & $1(2)$ & $33(4)$ & $264(34)$ \\
\hline Nasopharyngitis & $3(16)$ & $2(20)$ & $3(13)$ & $6(19)$ & $6(9)$ & $4(6)$ & $159(21)$ & $170(22)$ \\
\hline Headache & $3(16)$ & $3(30)$ & $4(17)$ & $7(23)$ & $9(13)$ & $8(12)$ & $129(17)$ & $133(17)$ \\
\hline Diarrhea & $1(5)$ & 0 & $4(17)$ & $8(26)$ & $3(4)$ & $4(6)$ & $83(11)$ & $107(14)$ \\
\hline Back pain & $3(16)$ & $1(10)$ & $4(17)$ & $4(13)$ & $6(9)$ & $8(12)$ & $90(12)$ & $94(12)$ \\
\hline Fatigue & $3(16)$ & $1(10)$ & $3(13)$ & $6(19)$ & $3(4)$ & $3(5)$ & $87(11)$ & $94(12)$ \\
\hline Nausea & $1(5)$ & $1(10)$ & $3(13)$ & $4(13)$ & $4(6)$ & $4(6)$ & $67(9)$ & $93(12)$ \\
\hline Proteinuria & $1(5)$ & $3(30)$ & $1(4)$ & $3(10)$ & $3(4)$ & $11(17)$ & $59(8)$ & $67(9)$ \\
\hline Pain in extremity & $1(5)$ & $1(10)$ & $4(17)$ & $3(10)$ & $4(6)$ & $7(11)$ & $58(8)$ & $58(8)$ \\
\hline Paraesthesia & $4(21)$ & $3(30)$ & $3(13)$ & $2(6)$ & $4(6)$ & $4(6)$ & $69(9)$ & $56(7)$ \\
\hline Albumin present in urine & $1(5)$ & $3(30)$ & 0 & 0 & $2(3)$ & $12(18)$ & $27(4)$ & $46(6)$ \\
\hline Hematuria & $1(5)$ & $2(20)$ & 0 & $1(3)$ & $5(7)$ & $5(8)$ & $34(4)$ & $33(4)$ \\
\hline Hypoesthesia & $3(16)$ & $4(40)$ & $2(9)$ & $2(6)$ & $5(7)$ & $1(2)$ & $48(6)$ & $31(4)$ \\
\hline Pyrexia & $1(5)$ & $1(10)$ & $1(4)$ & $1(3)$ & $17(24)$ & $12(18)$ & $40(5)$ & $27(4)$ \\
\hline Alopecia & $3(16)$ & $2(20)$ & $1(4)$ & 0 & $2(3)$ & $1(2)$ & $19(2)$ & $17(2)$ \\
\hline $\begin{array}{l}\text { No. of patients with any } \\
\text { serious } \mathrm{AE}^{\mathrm{c}}\end{array}$ & $3(16)$ & 0 & $5(22)$ & $3(10)$ & $26(37)$ & $21(32)$ & $165(21)$ & $135(18)$ \\
\hline $\begin{array}{l}\text { No. of patients with any AE } \\
\text { leading to treatment } \\
\text { discontinuation }\end{array}$ & $2(11)$ & $2(20)$ & $1(4)$ & $2(7)$ & $8(11)$ & $3(5)$ & $93(12)$ & $109(14)$ \\
\hline
\end{tabular}

$A E$ adverse event, $B I D$ twice daily, $M S$ multiple sclerosis

${ }^{a}$ DMF denotes delayed-release dimethyl fumarate (DMF; also known as gastro-resistant DMF)

b With the exception of multiple sclerosis relapse, these events are those reported by more than $10 \%$ of patients treated with DMF BID in any race subgroup analyzed, with an incidence that was at least $3 \%$ higher than in the placebo group, and occurring in at least 3 patients in any race subgroup. The events are listed by decreasing incidence among DMF-treated patients in the ITT population except for multiple sclerosis relapse

${ }^{c}$ No deaths occurred during the study or within 30 days of withdrawal in black, Hispanic, and Asian patients; there were two deaths reported in the ITT population: one in the DMF BID group (DEFINE) due to a bicycle accident 3 weeks after withdrawing from the study, and one in the placebo group (CONFIRM) due to stroke 
$49 \%(p<0.0001)$, in the risk of relapse by $43 \%$ $(p<0.0001)$, and in the risk of 12 -week CDP by $32 \%(p=0.0034)[22]$. For black patients, the corresponding relative risk reductions were 95\%, 93\%, and 47\%, respectively; in Hispanic patients the risk reductions were $69 \%, 45 \%$, and $83 \%$; and in Asian patients $36 \%, 34 \%$, and $29 \%$. Statistical significance was not achieved for any of the subgroups. No unexpected safety findings were observed in the three races analyzed, with AE profiles similar to those seen in the overall DEFINE and CONFIRM populations.

While the mean time since first MS diagnosis was broadly similar across groups, the mean age of patients with MS ranged from 31.7 (Hispanic patients) to 39.0 years (black patients) at study entry (Table 1). Other characteristics were generally well balanced across these subgroups and between the placebo and DMF groups, with the exception of the percentage of female patients with MS in the DMF and placebo treatment groups of the Asian study population (73\% versus $54 \%$, respectively).

One of the obvious confounders of evaluating the effects of treatment in particular ethnic or racial groups is geographic location. This is particularly true in MS, where geography has been shown to influence disease incidence [1]. The analysis of the clinical and MRI results of DEFINE and CONFIRM was adjusted for region, which was predefined on the basis of geography, type of health care system, and health care access. Region 1 was defined as USA; Region 2 as Western European countries (Belgium, France, Germany, Greece, Ireland, and Spain), Canada, Costa Rica, Australia, New Zealand, Israel, and South Africa; and Region 3 comprised Eastern European countries (Belarus, Bosnia and Herzegovina, Bulgaria, Croatia, Czech Republic, Estonia, Latvia, Macedonia, Moldova, Poland, Romania, Serbia, Slovakia, and Ukraine), India, Guatemala, and Mexico. In a post hoc analysis of the overall DEFINE and CONFIRM ITT population, the effects of DMF BID were consistent across regions [22].

The current integrated analysis was an appropriate means of exploring the efficacy and safety of DMF across racial subgroups of patients with MS, as it allowed for combination of data from studies with nearly identical designs that involved patients with similar characteristics to be pooled to create a more meaningful sample size. However, the numbers of black, Hispanic, and Asian patients with MS in the DEFINE and CONFIRM trials were still relatively small, resulting in limited statistical power to detect a treatment difference. Furthermore, the post hoc nature of this analysis means that its findings can only be viewed as hypothesis generating and that results should be interpreted with caution. This is one of many subgroup analyses performed on patients in DEFINE and CONFIRM [22], and has the potential for multiplicity problems (inflated false-positive rate).

The findings of the current study add to those from other post hoc analyses of integrated data from DEFINE and CONFIRM, which have suggested that DMF has a strong and consistent effect on clinical outcomes across a wide range of patients $[22,28]$, health-related quality of life benefits [29], and a rapid onset of action [30]. In the USA, non-white races represent more than $27 \%$ of the population [18] but often make up less than $7 \%$ of the MS clinical trials population [1]. Thus, there is a need for more robust and prospective inclusion of diverse ethnic groups in clinical studies, which will be achieved by modifying future study designs and engaging in specific outreach initiatives with currently underrepresented key patient groups. Larger prospective studies are warranted to further investigate the effect of DMF in these populations.

\section{CONCLUSION}

This post hoc analysis suggests that DMF may be an efficacious treatment with a favorable benefit-risk profile in black, Hispanic, and Asian patients with RRMS. The results on clinical efficacy and safety measures in these populations are consistent with those reported in the overall population in DEFINE and CONFIRM. More studies are needed to further characterize differences in MS presentation across various ethnic or racial groups and how these may affect treatment outcomes. Such studies will contribute to improvement in the 
understanding of the disease landscape, guide treatment enhancements, and ultimately lead to better quality care in ethnically diverse patient populations.

\section{ACKNOWLEDGEMENTS}

This study was sponsored by Biogen. Biogen provided funding for the article processing charges and provided funding for medical writing support in the development of this paper; Juliette Allport, M.Sc., from Complete Medical Communications and Ana Antaloae, Ph.D., from Excel Scientific Solutions wrote the first draft of the manuscript based on input from authors, and Elizabeth Cassell from Excel Scientific Solutions copyedited and styled the manuscript per journal requirements. Biogen reviewed and provided feedback on the paper to the authors. The authors meet the International Committee of Medical Journal Editors (ICMJE) criteria for authorship for this manuscript, had full editorial control of the paper, and provided their final approval of all content and for this version to be published. All authors had full access to all of the data in this study and take complete responsibility for the integrity of the data and accuracy of the data analysis.

Disclosures. Robert J. Fox reports consultant fees from Allozyne, Avanir, Biogen, Novartis, Questcor, Teva, and Xenoport; advisory committees for Biogen and Novartis; and research grant funding from Novartis. Ralf Gold reports honoraria from Bayer HealthCare, Biogen, Merck Serono, Novartis, and Teva Neuroscience; research support from Bayer HealthCare, Biogen, Merck Serono, Novartis, and Teva Neuroscience; and compensation from Sage for serving as editor of Therapeutic Advances in Neurological Disorders. J. Theodore Phillips reports honoraria from Acorda, Biogen, EMD Serono, Genentech, and Genzyme. Macaulay Okwuokenye reports that he is an employee of and holds stock/stock options in Biogen. Annie Zhang reports that the majority of the work was performed whilst an employee of Biogen, Cambridge, MA; her current affiliation is with
Apellis Pharmaceutical, Inc. Jing L. Marantz reports that the majority of the work was performed whilst an employee of Biogen, Cambridge, MA; her current affiliation is with Alexion Pharmaceuticals, New Haven, CT.

Compliance with Ethics Guidelines. All procedures followed were in accordance with the ethical standards of the responsible committee on human experimentation (institutional and national) and with the Helsinki Declaration of 1964, as revised in 2013. Informed consent was obtained from all patients for being included in the study. The DEFINE and CONFIRM studies were approved by central and local ethics committees and were conducted in accordance with the International Conference on Harmonisation's guidelines for Good Clinical Practice and the Declaration of Helsinki. All patients provided re-consent in the event of confirmed relapse or disability progression.

Data Availability. The datasets generated and/or analyzed during the current study are not publicly available because of patient confidentiality and compliance reasons, but are available from the corresponding author on reasonable request.

Open Access. This article is distributed under the terms of the Creative Commons Attribution-NonCommercial 4.0 International License (http://creativecommons.org/licenses/ by-nc/4.0/), which permits any noncommercial use, distribution, and reproduction in any medium, provided you give appropriate credit to the original author(s) and the source, provide a link to the Creative Commons license, and indicate if changes were made.

\section{REFERENCES}

1. Rosati G. The prevalence of multiple sclerosis in the world: an update. Neurol Sci. 2001;22:117-39.

2. Langer-Gould A, Brara SM, Beaber BE, Zhang JL. Incidence of multiple sclerosis in multiple racial and ethnic groups. Neurology. 2013;80:1734-9. 
3. Khan O, Williams MJ, Amezcua L, Javed A, Larsen KE, Smrtka JM. Multiple sclerosis in US minority populations: clinical practice insights. Neurol Clin Pract. 2015;5:132-42.

4. Kister I, Chamot E, Bacon JH, et al. Rapid disease course in African Americans with multiple sclerosis. Neurology. 2010;75:217-23.

5. Weinstock-Guttman B, Jacobs LD, Brownscheidle $\mathrm{CM}$, et al. Multiple sclerosis characteristics in African American patients in the New York State Multiple Sclerosis Consortium. Mult Scler. 2003;9:293-8.

6. Buchanan RJ, Zuniga MA, Carrillo-Zuniga G, et al. Comparisons of Latinos, African Americans, and Caucasians with multiple sclerosis. Ethn Dis. 2010;20:451-7.

7. Cree BA, Khan O, Bourdette D, et al. Clinical characteristics of African Americans vs Caucasian Americans with multiple sclerosis. Neurology. 2004;63:2039-45.

8. Chong HT, Li PCK, Ong B, et al. Long term outcome of multiple sclerosis in Asia. Neurol Asia. 2006;11:49-53.

9. West T, Wyatt M, High A, Bostrom A, Waubant E. Are initial demyelinating event recovery and time to second event under differential control? Neurology. 2006;67:809-13.

10. Avasarala J. Inadequacy of clinical trial designs and data to control for the confounding impact of race/ ethnicity in response to treatment in multiple sclerosis. JAMA Neurol. 2014;71:943-4.

11. Humes KR, Jones NA, Ramirez RR. Overview of race and Hispanic origin: 2010. 2011. https://www. census.gov/prod/cen2010/briefs/c2010br-02.pdf. Accessed 14 July 2017.

12. Cree BA, Al-Sabbagh A, Bennett R, Goodin D. Response to interferon beta-1a treatment in African American multiple sclerosis patients. Arch Neurol. 2005;62:1681-3.

13. Klineova S, Nicholas J, Walker A. Response to disease modifying therapies in African Americans with multiple sclerosis. Ethn Dis. 2012;22:221-5.

14. Chinea Martinez AR, Correale J, Coyle PK, Meng X, Tenenbaum N. Efficacy and safety of fingolimod in Hispanic patients with multiple sclerosis: pooled clinical trial analyses. Adv Ther. 2014;31:1072-81.

15. Cree BA, Stuart WH, Tornatore CS, Jeffery DR, Pace $\mathrm{AL}$, Cha $\mathrm{CH}$. Efficacy of natalizumab therapy in patients of African descent with relapsing multiple sclerosis: analysis of AFFIRM and SENTINEL data. Arch Neurol. 2011;68:464-8.

16. Biogen. Tecfidera (prescribing information) (online). Cambridge, MA 02142. 2016. http://www. tecfidera.com/pdfs/full-prescribing-information.pdf. Accessed 18 Jul 2016.

17. Biogen Australia. Product information. Tecfidera ${ }^{\circledR}$ [dimethyl fumarate (DMF)] (online). 2013. http:// www.tga.gov.au/sites/default/files/auspar-dimethylfumarate-131022-pi.docx. Accessed 18 Jul 2016.

18. Biogen. Dimethyl fumarate package leaflet: information for the patient (online). 2015. http://www. medicines.org.uk/emc/PIL.28650.latest.pdf. Accessed 18 Jul 2016.

19. Biogen Canada. Product monograph. Tecfidera (online). 2015. http://www.biogen.ca/content/ dam/corporate/en_CA/pdfs/products/TECFIDERA/1TECFIDERA-PM-2015-01-29-E.pdf. Accessed 18 Jul 2016.

20. Fox RJ, Miller DH, Phillips JT, et al. Placebo-controlled phase 3 study of oral BG-12 or glatiramer in multiple sclerosis. N Engl J Med. 2012;367:1087-97.

21. Gold R, Kappos L, Arnold DL, et al. Placebo-controlled phase 3 study of oral BG-12 for relapsing multiple sclerosis. N Engl J Med. 2012;367:1098-107.

22. Viglietta V, Miller D, Bar-Or A, et al. Efficacy of delayed-release dimethyl fumarate in relapsing-remitting multiple sclerosis: integrated analysis of the phase 3 trials. Ann Clin Transl Neurol. 2015;2:103-18.

23. Kurtzke JF. Rating neurologic impairment in multiple sclerosis: an Expanded Disability Status Scale (EDSS). Neurology. 1983;33:1444-52.

24. Polman CH, Reingold SC, Edan G, et al. Diagnostic criteria for multiple sclerosis: 2005 revisions to the "McDonald Criteria". Ann Neurol. 2005;58:840-6.

25. Firth D. Bias reduction of maximum likelihood estimates. Biometrika. 1993;1:27-38.

26. Heinze G, Schemper M. A solution to the problem of monotone likelihood in Cox regression. Biometrics. 2001;57:114-9.

27. Zhovtis Ryerson L, Green R, Confident G, et al. Efficacy and tolerability of dimethyl fumarate in white-, African- and Hispanic-Americans with multiple sclerosis. Ther Adv Neurol Disord. 2016;9:454-61.

28. Gold R, Giovannoni G, Phillips JT, et al. Efficacy and safety of delayed-release dimethyl fumarate in patients newly diagnosed with relapsing-remitting 
multiple sclerosis (RRMS). Mult Scler. 2015;21:57-66.

29. Kita M, Fox RJ, Gold R, et al. Effects of delayed-release dimethyl fumarate (DMF) on health-related quality of life in patients with relapsing-remitting multiple sclerosis: an integrated analysis of the phase 3 DEFINE and CONFIRM studies. Clin Ther. 2014;36:1958-71.
30. Kappos L, Giovannoni G, Gold R, et al. Time course of clinical and neuroradiological effects of delayed-release dimethyl fumarate in multiple sclerosis. Eur J Neurol. 2015;22:664-71. 